\title{
Sु \\ Coherence of single spins coupled to a nuclear spin bath of varying density
}

\author{
N. Mizuochi,,${ }^{1,2}$ P. Neumann, ${ }^{3}$ F. Rempp, ${ }^{3}$ J. Beck, ${ }^{3}$ V. Jacques, ${ }^{3}$ P. Siyushev, ${ }^{3}$ K. Nakamura, ${ }^{4}$ D. J. Twitchen, ${ }^{5}$ \\ H. Watanabe, ${ }^{6}$ S. Yamasaki, ${ }^{7}$ F. Jelezko, ${ }^{3}$ and J. Wrachtrup ${ }^{3}$ \\ ${ }^{1}$ Graduate School of Library, Information \& Media Studies, University of Tsukuba, 1-2 Kasuga, 305-8550, Japan \\ ${ }^{2}$ PRESTO JST, 4-1-8 Honcho, Kawaguchi, Saitama 332-0012, Japan \\ 3. Physikalisches Institut, Universität Stuttgart, Pfaffenwaldring 57, D-70550 Stuttgart, Germany \\ ${ }^{4}$ Tokyo Gas Co., Ltd., 3-13-1 Minamisenju, Tokyo 116-0003, Japan \\ ${ }^{5}$ Element Six Ltd., King's Ride Park, Ascot, Berkshire SL5 8BP, United Kingdom \\ ${ }^{6}$ Diamond Research Center, AIST, Tsukuba Central 2, Tsukuba 305-8568, Japan \\ ${ }^{7}$ Nanotechnology Research Institute, AIST, Tsukuba Central 2, Tsukuba 305-8568, Japan
}

(Received 19 June 2009; published 13 July 2009)

\begin{abstract}
The dynamics of single electron and nuclear spins in a diamond lattice with different ${ }^{13} \mathrm{C}$ nuclear spin concentration is investigated. It is shown that coherent control of up to three individual nuclei in a dense nuclear spin cluster is feasible. The free-induction decays of nuclear spin Bell states and single nuclear coherences among ${ }^{13} \mathrm{C}$ nuclear spins are compared and analyzed. Reduction in a free-induction-decay time $T_{2}^{*}$ and a coherence time $T_{2}$ upon increase in nuclear spin concentration has been found. For pure diamond, $T_{2}^{*}$ as long as $30 \mu \mathrm{s}$ and $T_{2}$ of up to $0.65 \mathrm{~ms}$ for the electron spin has been observed. The ${ }^{13} \mathrm{C}$ concentration dependence of $T_{2}^{*}$ is explained by Fermi contact and dipolar interactions with nuclei in the lattice. It has been found that $T_{2}$ decreases approximately as $1 / n$, where $n$ is ${ }^{13} \mathrm{C}$ concentration, which corresponds to the reported theoretical line of $T_{2}$ for an electron spin interacting with a nuclear spin bath.
\end{abstract}

DOI: 10.1103/PhysRevB.80.041201

Defect centers in diamond have attracted considerable interest recently owing to their application for quantum information processing, communication, and metrology, ${ }^{1-7}$ especially the nitrogen-vacancy (NV) center, with its strong and spin-dependant optical transitions that allows for single spin readout and exquisite coherent control, which is crucial for quantum information applications. ${ }^{1-5}$ Owing to the high Debye temperature of diamond and weak coupling to acoustic phonons NV electron spins show long coherence time. It was, e.g., proposed to build small quantum registers by exploiting the interaction between the electron spin and a small number of nuclear spins in the immediate vicinity. Five-qubit would be sufficient to perform all functions necessary for a node in a defect center based quantum repeater node. ${ }^{4,5} \mathrm{Up}$ to now coherent control, swapping of coherence, and even entanglement between up to two nuclei and the electron spin were demonstrated. ${ }^{3}$ To increase the size of the quantum register, more nuclei need to be coupled to the electron spin. The approach taken here is to increase the concentration of paramagnetic ${ }^{13} \mathrm{C}$ nuclei in the lattice. We systematically demonstrate coherent control of up to three nuclear spins being coupled to an NV center electron spin in ${ }^{13} \mathrm{C}$ isotopically enriched crystals, notwithstanding the fact that the electron decoherence time $T_{2}$ linearly scales with the ${ }^{13} \mathrm{C}$ concentration. Furthermore, our experiments provide experimental insight into long studied problem of single central spin coupled to a paramagnetic environment. ${ }^{8-10}$ Owing to possibility of addressing individual electron spins in matrix with adjustable nuclear spin content we show the transition from diluted to dense spin bath (the situation relevant for spins in GaAs quantum dots).

The quantum system used in the present work is the negatively charged NV center in diamond, which comprises a substitutional nitrogen atom with an adjacent vacancy. [Fig. 1(h)] The electron ground state of it is a spin triplet. Upon
PACS number(s): 76.30.Mi, 03.65.Yz, 03.67.-a, 71.55.Cn

optical excitation the NV center shows strong fluorescence allowing it to be observed on an individual basis by confocal microscopy. The fluorescence intensity of the defect is spin dependent owing to spin selective relaxation via singlet state, which allows optical read out of the single ESR (Ref. 11) and an efficient electron and nuclear initialization at room temperature. Microwaves (MWs) and radio frequency (RF) fields are used for coherent manipulation of single electron and nuclear spins using conventional ESR/NMR techniques.
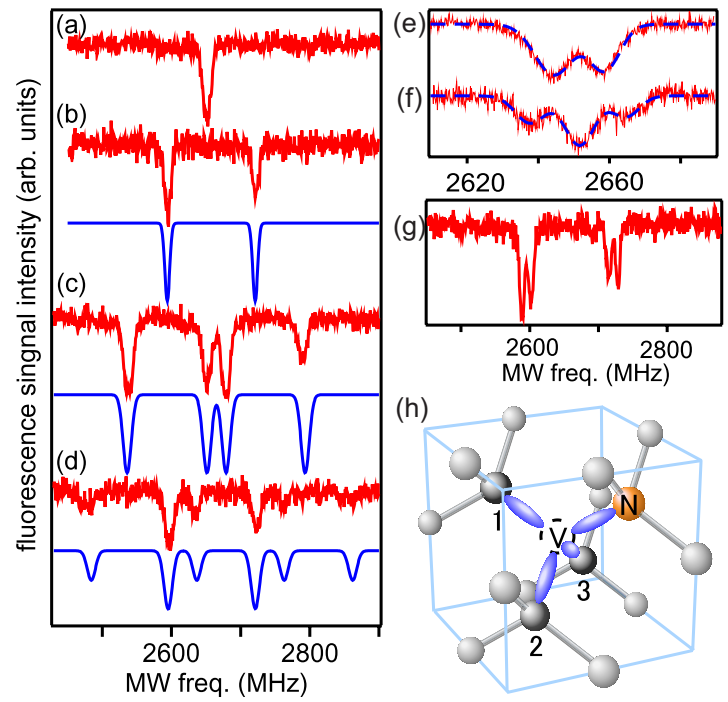

FIG. 1. (Color online) Electron-spin resonance (ESR) spectra of single NVs with (a) zero, (b) one, (c) two, and (d) three ${ }^{13} \mathrm{C}$ in the first shell. Lower solid lines (blue) in (b-d) are simulation spectra. ESR spectra with (e) one and (f) two ${ }^{13} \mathrm{C}$ in the third shell, and $(\mathrm{g})$ one ${ }^{13} \mathrm{C}$ in the first shell and one ${ }^{13} \mathrm{C}$ in the third shell. Blue dashed lines are fitting lines with Gaussian. (h) Atomic structure of NV center. The numbers 1,2 , and 3 mark $\mathrm{C}$ in the first shell. 
Measurement is carried out at $\sim 20{ }^{\circ} \mathrm{C}$. Diamond enables for the unique opportunity to control the concentration of paramagnetic nuclear spins. The most abundant ${ }^{12} \mathrm{C}$ has zero nuclear spin. The concentration of ${ }^{13} \mathrm{C}$ nuclear spins ( $I$ $=1 / 2$ ) can be adjusted to the suitable value by controlling the isotopic content of ${ }^{13} \mathrm{C}$ in the growth medium. Two types of synthetic diamonds were used in this study. Crystals with $0.35 \%, 1.1 \%, 8.4 \%$, and $20.7 \%{ }^{13} \mathrm{C}$ concentrations were synthesized by a MW plasma-assisted homoepitaxial chemical vapor deposition (CVD) (Refs. 12 and 13) technique with ${ }^{13} \mathrm{CH}_{4}$ or ${ }^{12} \mathrm{CH}_{4}$ gases. The $0.03 \%{ }^{13} \mathrm{C}$ and $\sim 100 \%{ }^{13} \mathrm{C}$ enriched diamonds were synthesized by high-pressure hightemperature (HPHT) method using getters preventing incorporation of nitrogen into lattice. In all samples the concentration of paramagnetic impurity measured by ESR was under detection limit (below $1 \mathrm{ppb}$ ) except $0.03 \%$ and $100 \%{ }^{13} \mathrm{C}$ diamond where the concentration of nitrogen was at 1 ppm level. ${ }^{14}$

$\mathrm{NV}$ centers with different numbers of ${ }^{13} \mathrm{C}$ atoms in the immediate vicinity of the electron spin have been investigated in $8.4 \%{ }^{13} \mathrm{C}$ diamond. Figures $1(\mathrm{a})-1(\mathrm{~d})$ show single ESR spectra indicating the hyperfine coupling (HFC) of the electron spin to zero, one, two, and three nuclear spins in the first shell [Fig. 1(h)]. A magnetic field of $\sim 83 \mathrm{G}$ was oriented along the NV axis ([111]-axis). To characterize the spin quantum states associated with the transitions in Figs. 1(b)-1(d), the spectra were simulated by exact diagonalization of the spin Hamiltonian

$$
H=g_{e} \beta_{e} \widetilde{S} B+\widetilde{S} D S+\left(\widetilde{S} A I_{i}-g_{n} \beta_{n} I_{i} B\right) .
$$

Here the electron spin $S=1$ and ${ }^{13} \mathrm{C}$ nuclear spins in first shell are taken into account. $\beta_{e}$ is the Bohr and $\beta_{n}$ is the nuclear magneton, respectively. Reported values for zerofield splitting (ZFS) parameter $(|D|=2.87 \mathrm{GHz}),{ }^{15}$ isotropic electron and nuclear Zeeman $g$ values $\left(g_{e}=2.0028, g_{n}\right.$ $=1.40483)$, and HFC parameters of $A_{\|}=205 \mathrm{MHz}$ and $A_{\perp}$ $=123 \mathrm{MHz}$ (Ref. 16) with angle of $106^{\circ}$ between principal axes of ZFS and HFC yield precise fits of the experimental spectra. The small splittings in the central signals of the spectra in Figs. 1(c) and 1(d) are explained by a second-order perturbation approach. ${ }^{3}$ The smaller amplitudes in higher frequency are due to absorption of MW by wire on the sample.

In the ESR spectra of $8.4 \%{ }^{13} \mathrm{C}$ diamond, basically two types of couplings are immediately visible [Figs. 1(b)-1(g)]: those around $130 \mathrm{MHz}$ originating from first shell ${ }^{13} \mathrm{C}$ and those around $14 \mathrm{MHz}$. In $C_{3 v}$ symmetry, the number of equivalent atoms in close shells around $\mathrm{NV}$ is 3 or 6 . In recent theoretical study, ${ }^{17}$ the $14 \mathrm{MHz}$ splittings are assigned to ${ }^{13} \mathrm{C}$ at 3 and 6 equivalent sites in the third shell (see video in Ref. 18). From measuring more than 250 individual centers and comparing the probability to find the $14 \mathrm{MHz}$ splitting with the one predicted from theory we assign this splitting to nuclei in the third shell. ${ }^{18}$

Individual nuclei in the spin cluster around the electron are addressed via their particular NMR frequency. Given the increase in spectral density apparent from Fig. 1 one might wonder how far individual nuclei remain addressable. However, coherent control even in dense spin clusters remains
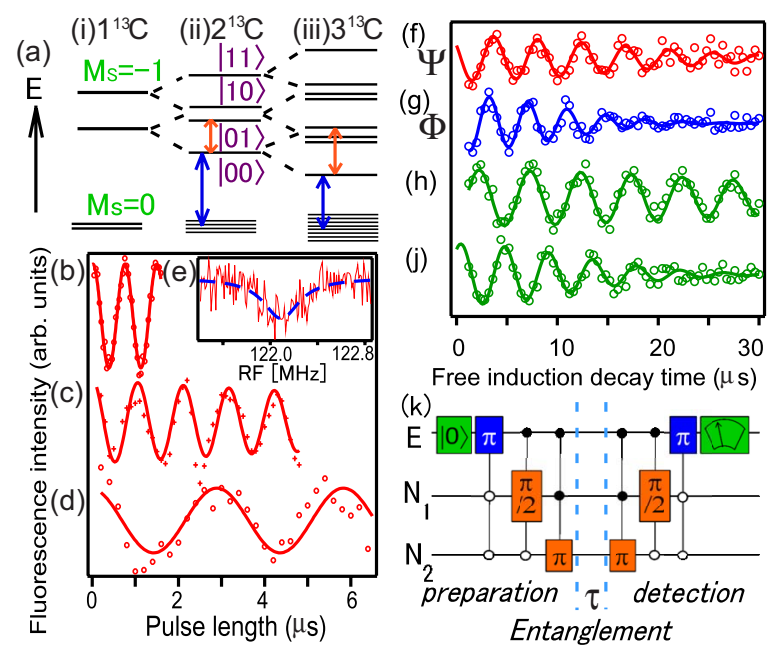

FIG. 2. (Color online) (a) Energy levels for nuclear spins in $M_{S}=-1$. Rabi oscillation of single ${ }^{13} \mathrm{C}$ in the first shell of neighbors around the NV with (b) two ${ }^{13} \mathrm{C}$ in the first shell and (c) three ${ }^{13} \mathrm{C}$ in the first shell. (d) Rabi oscillation of single ${ }^{13} \mathrm{C}$ in the third shell with one ${ }^{13} \mathrm{C}$ in the first shell and one ${ }^{13} \mathrm{C}$ in the third shell. The pulse sequence is $\pi(\mathrm{MW})-\mathrm{Rabi}(\mathrm{RF})-\pi(\mathrm{MW})$ (Ref. 1). The ESR transitions of the MW $\pi$ pulse are those at lowest frequency in Figs. 1(c), 1(d), and 1(g) and are indicated by dark gray (blue) arrows in (a). The NMR transitions of the RF pulse are indicated by light gray (orange) arrows in (a). The recording the data in (b)-(d) required about $20 \mathrm{~min}$ of averaging. (e) Electron nuclear double resonance (ENDOR) spectrum of ${ }^{13} \mathrm{C}$ at first shell in (b) with fitted Gaussian line (blue dashed line) by the pulse sequence of $\pi(\mathrm{MW})-\pi(\mathrm{RF})-\pi(\mathrm{MW})$. Free-induction decay (FID) of (f) $\Psi^{-},(\mathrm{g})$ $\Phi^{-}$, and (h) nuclear coherence between $|00\rangle \leftrightarrow|10\rangle$ and (j) $|00\rangle \leftrightarrow|01\rangle$. (k) Pulse sequence for $\Phi^{-}$generation and detection between two nuclear spins. $E$ and $N_{1,2}$ marks the electron and the two nuclear spins, respectively. Spin selective pulses are represented by squares, operating on a target qubit. Vertical lines represent logical connections. The control qubit state $|1\rangle$ and the state $|0\rangle$ are displayed as filled $(\bullet)$ and open $(\bigcirc)$ circles. For example, $(\bigcirc)$ indicates that the pulse is applied to the target qubit if the quantum state of the controlling qubit is $|0\rangle$.

feasible as demonstrated in Fig. 2. Even in cases where there are three ${ }^{13} \mathrm{C}$ in the first shell, i.e., in total four qubits, Rabi nutations of single nuclear spins can be driven by an additional RF as shown in Fig. 2(c). That is because even multiple RF transition frequencies originating from nuclei at equivalent positions split due to higher order HFC contributions as shown above. The selectivity is not limited to the relatively large splitting in the first shell but can be applied to ${ }^{13} \mathrm{C}$ nuclear spins in the third shell. A spectrum of single $\mathrm{NV}$, which has one ${ }^{13} \mathrm{C}$ in the first shell and one ${ }^{13} \mathrm{C}$ in third shell, is shown in Fig. 1(g). We labeled the four nuclear spin states as $|00\rangle,|01\rangle,|10\rangle$, and $|11\rangle$ as shown in Fig. 2(a). Rabi oscillations between $|00\rangle$ and $|01\rangle$ could be observed as shown in Fig. 2(d). To obtain a similar Rabi frequency for ${ }^{13} \mathrm{C}$ nuclear spins in the third shell as for those in the first shell, about $1 \times 10^{2}$ times higher RF power was necessary. This can be interpreted mainly by hyperfine enhancement,${ }^{19}$ which predicts that Rabi frequency is almost proportional to HFC and the square root of the RF power. A ten times smaller $\mathrm{HFC}$ in the third shell supports this interpretation. A figure of 
merit that characterizes the quality of coherent control was derived by swapping quantum states among individual nuclei. It was estimated by transferring polarization back to a detectable electron-spin state that $82 \pm 5 \%$ of polarization was transferred from $|00\rangle$ to $|01\rangle$ for ${ }^{13} \mathrm{C}$ nuclear spins in the third shell.

We are now in a position to engineer simple quantum states in the spin cluster around the electrons spin. Bell states $\Phi^{ \pm}=1 / \sqrt{2}(|00\rangle \pm|11\rangle)$ and $\Psi^{ \pm}=1 / \sqrt{2}(|01\rangle \pm|10\rangle)$ can be generated from the four effective nuclear spin states in Fig. 2(a). In the present case they were prepared from two ${ }^{13} \mathrm{C}$ spins at the first shell. The procedure follows previous studies $^{3,20}$ and is schematically shown for $\Phi^{-}$in Fig. $2(\mathrm{k})$. After its generation, $\Phi^{-}$shows a FID, which is made visible with an entanglement detector sequence [Fig. 2(k)]. In $8.4 \%$ ${ }^{13} \mathrm{C}$ diamond, the free-induction-decay times $T_{2}^{*}$ of $\Psi^{-}\left(T_{2(\Psi)}^{*}\right)$ and $\Phi^{-}\left(T_{2(\Phi)}^{*}\right)$ were estimated to be $22.0 \pm 3.0 \mu \mathrm{s}$ and $13.3 \pm 1.1 \mu \mathrm{s}$, [see Figs. 2(f) and $2(\mathrm{~g})]$ respectively, by fitting with $\exp \left[-\left(t / T_{2}^{*}\right)^{2}\right] \cos (\Delta \omega t),{ }^{8}$ where $\Delta \omega$ is the detuned frequency of FID. As expected from the view point of decoherence free subspaces, ${ }^{21}$ a longer $T_{2(\Psi)}^{*}$ compared to that of $T_{2(\Phi)}^{*}$ is observed.

The difference among $T_{2(\Psi)}^{*}$ and $T_{2(\Phi)}^{*}$ is best analyzed when compared with $T_{2}^{*}$ of a nuclear quantum coherence among states $|00\rangle \leftrightarrow|10\rangle$ and $|00\rangle \leftrightarrow|01\rangle$. Those coherences are labeled as single-quantum coherences SQ1 and SQ2, respectively. Their $T_{2}^{*}$ are measured to be $T_{2(S Q 1)}^{*}$ $=41.1 \pm 3.1 \mu \mathrm{s}$ and $T_{2(S Q 2)}^{*}=15.8 \pm 1.4 \mu \mathrm{s}$, respectively [Figs. 2(h) and 2(j)]. The difference of $T_{2(S Q 1)}^{*}$ and $T_{2(S Q 2)}^{*}$ might be caused by a spatially inhomogeneous magnetic noise around the defect caused by an inhomogeneous distribution of ${ }^{13} \mathrm{C}$ around the two ${ }^{13} \mathrm{C}$ in the first shell. Each spin-spin interaction between nuclear spin $k$ surrounding the two ${ }^{13} \mathrm{C}$ in the first shell with quantum numbers $m_{I 1}$ and $m_{I 2}$ causes oscillation given by $\sum_{k} \exp \left[-i\left(\Delta \omega_{1} m_{I 1}+\Delta \omega_{2} m_{I 2}\right) t\right] .{ }^{22}$ Here $\Delta \omega_{1}$ and $\Delta \omega_{2}$ are spin-spin interaction frequencies of the two ${ }^{13} \mathrm{C}$ in the first shell due to surrounding ${ }^{13} \mathrm{C}$ nuclear spins. This implies that $T_{2(\Psi)}^{*}$ and $T_{2(\Phi)}^{*}$ can be approximated by $1 / T_{2(\Psi)}^{*}=\left|1 / T_{2(S Q 1)}^{*}-1 / T_{2(S Q 2)}^{*}\right|$ and $1 / T_{2(\Phi)}^{*}=1 / T_{2(S Q 1)}^{*}$ $+1 / T_{2(S Q 2)}^{*}$, respectively. Inserting the measured values for $1 / T_{2(S Q 1,2)}^{*}$, the results are $T_{2 \text { calc. }(\Psi)}^{*}=25.8_{-4.3}^{+5.7} \mu \mathrm{s}$ and $T_{2 \text { calc. }(\Phi)}^{*}=11.4_{-0.9}^{+1.0} \mu \mathrm{s}$, respectively, in good correspondence with measured values.

Besides the effect of the nuclear spin bath on individual ${ }^{13} \mathrm{C}$ spins the static interaction between the single $\mathrm{NV}$ electron spin and its environment for different ${ }^{13} \mathrm{C}$ concentrations was investigated by measuring $T_{2}^{*}$, i.e., the inhomogeneous ESR linewidth. It was observed that $T_{2}^{*}$ increases, i.e., the linewidth narrows, with decreasing ${ }^{13} \mathrm{C}$ concentration as shown in Fig. 3. In $0.03 \%{ }^{13} \mathrm{C}$ diamond, an extremely long $T_{2}^{*}$ of $30 \mu \mathrm{s}$ was found (see Fig. 3 ). In the low ${ }^{13} \mathrm{C}$ concentration region $(\leqq 1.1 \%$ ), the linewidth $W$ (full width at half maximum) is derived from $T_{2}^{*}$ by $W=2 \sqrt{\ln 2} / \pi T_{2}^{*}$. The corresponding $18 \mathrm{kHz}$ linewidth is the narrowest ever observed for an electron spin in a solid material. In the high ${ }^{13} \mathrm{C}$ concentration region $(>1.1 \%)$, the linewidth is derived from fitting the ESR line of a single NV with a Gaussian lineshape. Average values are plotted as squares in Fig. 3(e).

A likely cause for the inhomogeneous ESR linewidth is
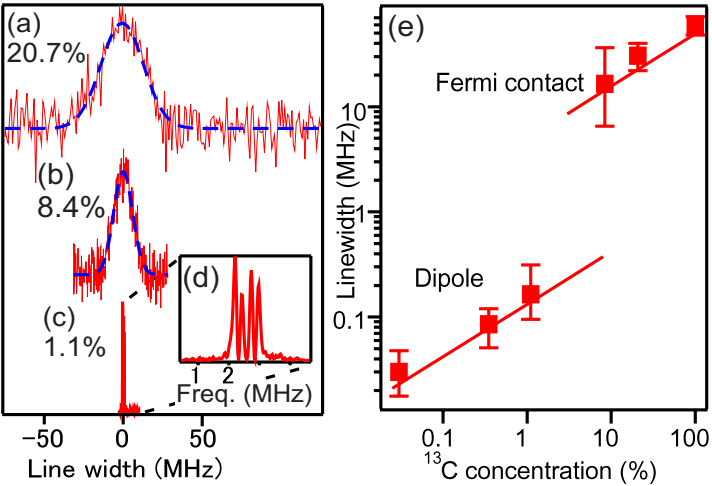

FIG. 3. (Color online) ESR spectra of single NV in (a) $20.7 \%$ and (b) $8.4 \%{ }^{13} \mathrm{C}$ diamond with fitted Gaussian lines (blue dashed lines). (c) Fourier-transformed spectrum of FID of $1.1 \%{ }^{13} \mathrm{C}$ diamond shown in (d) on expanded frequency axis. The splitting is due to $\mathrm{HFC}$ of distant ${ }^{13} \mathrm{C}$ nuclear spins. The hyperfine splitting to $N$ is not visible in this spectrum due to polarization of the $\mathrm{N}$ nuclear spin (Ref. 28). (e) Dependence of Inhomogeneous linewidth on ${ }^{13} \mathrm{C}$ concentration. The error bars indicate the distributions measured.

HFC to ${ }^{13} \mathrm{C}$ nuclear spins. In, e.g., Si, the dependence of the inhomogeneous linewidth of $\mathrm{P}$ donors on ${ }^{29} \mathrm{Si}$ is well fitted by the isotropic HFC $\left(a_{l}\right)$ due to Fermi contact interaction with ${ }^{29} \mathrm{Si}$ nuclear spins with a concentration $(f), W$ $=2 \sqrt{2 \ln 2}\left[f \Sigma_{l}\left(a_{l} / 2\right)^{2}\right]^{1 / 2} \cdot{ }^{23,24}$ The sum runs over all nuclear spin sites $l$. In Fig. 3(e), the solid line for high ${ }^{13} \mathrm{C}$ concentrations is calculated by summing only over all the nine sites in the third shell with $a_{l}=14 \mathrm{MHz}$ (see above for assignment of sites and HFC constants). It should be noted that contributions from ${ }^{13} \mathrm{C}$ in the first shell were not considered in the linewidth calculations because they contribute to an observable splitting but not to the linewidth. As seen from Fig. 3(e), it fits the experimental results well for high ${ }^{13} \mathrm{C}$ concentration.

For lower ${ }^{13} \mathrm{C}$ concentration, experimental data deviate from this behavior. This is due to the fact that the probability that any ${ }^{13} \mathrm{C}$ is located close to the $\mathrm{NV}$ center is getting small upon reduction in ${ }^{13} \mathrm{C}$ concentration. Furthermore, the unpaired electron-spin density rapidly decreases with distance from the three dangling bonds around the vacancy. This is known from the HFC parameters, ${ }^{16,17}$ which indicates that almost $100 \%$ spin density is localized on the $\mathrm{C}$ sites in the first and the third shells. That is why in this situation the most prominent contribution to the inhomogeneous linewidth is the weaker dipole-dipole interaction between electron spin and ${ }^{13} \mathrm{C}$ nuclear spin at distant sites. The lower line in Fig. $3(\mathrm{e})$ is the linewidth

$$
W=\sqrt{\left(\mu_{0} \mu_{e} \mu_{n} g_{e} g_{n} / 4 \pi h\right)^{2}\left(3.195 \times 10^{46} n\right)},
$$

calculated from the second moment ${ }^{25}$ with more than 3000 lattice sites for each ${ }^{13} \mathrm{C}$ concentration $(n)$. Contributions from ${ }^{13} \mathrm{C}$ in the first and second shells are not considered. As seen from Fig. 3(e), $W$ fits the experimental results in the low ${ }^{13} \mathrm{C}$ concentration $(\leqq 1 \%)$ quite well. Obviously at low ${ }^{13} \mathrm{C}$ concentration the linewidth is dominated by dipole-dipole interaction. 


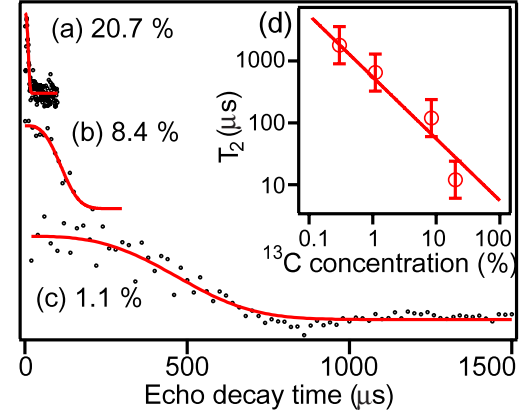

FIG. 4. (Color online) Echo decays of electron spin in (a) $20.7 \%$, (b) $8.4 \%$, and (c) $1.1 \%{ }^{13} \mathrm{C}$ diamond. The MW pulse sequence is $\pi / 2-\tau-\pi-\tau-\pi / 2$ where $\tau$ is delay (Ref. 8). Solid (red) lines are curves fitted with $\exp \left[-\left(t / T_{2}\right)^{3}\right]$. (d) Plot of $T_{2}$ over ${ }^{13} \mathrm{C}$ concentration $n$. The solid (red) line is fitted with a $1 / n$ dependence.

The dephasing time $T_{2}$ of the electron spin is measured by two pulse Hahn echo decay curves [Figs. 4(a)-4(c)]. We analyzed $T_{2}$ of the diamond made by CVD and excluded the $0.03 \%$ and $100 \%{ }^{13} \mathrm{C}$ diamond made by HPHT because paramagnetic impurities could not be suppressed in HPHT. In the $1.1 \%{ }^{13} \mathrm{C}$ diamond, a $T_{2}$ of $0.65 \mathrm{~ms}$ was found, which is the longest $T_{2}$ in diamond with natural abundance of ${ }^{13} \mathrm{C}$ measured so far. For the lower ${ }^{13} \mathrm{C}$ concentration of $0.3 \%$ an even longer $T_{2}$ of $1.8 \mathrm{~ms}$ was reported recently and added for the analysis. ${ }^{26} T_{2}$ is found to be inversely proportional to the ${ }^{13} \mathrm{C}$ concentration as plotted in Fig. 4(d).

In a theoretical analysis of $T_{2}$ by the disjoint cluster approach, ${ }^{5}$ the relationship of $T_{2} \sim\left(\bar{C} A_{c}\right)^{-1 / 2}$ is proposed, where $\bar{C}$ is the averaged nuclear-nuclear dipolar interaction in the bath and $A_{c}$ is some characteristic value for the electron-nuclear dipolar interaction. Since both interactions scale linearly in ${ }^{13} \mathrm{C}$ concentration $(n), T_{2}$ decreases approximately as $1 / n$ in this model. The fitted line to the data shown in Fig. 4(d) supports this inverse proportionality and fits our data. Note that our data also fit the values calculated in (Ref. 5) within $30 \%$ deviation.

In conclusion, coherent control of up to three individual nuclei in a dense nuclear spin cluster is demonstrated. The ${ }^{13} \mathrm{C}$ concentration dependence of $T_{2}^{*}$ and $T_{2}$ of electron spin point toward ${ }^{13} \mathrm{C}$ nuclei as the main cause for dephasing in otherwise clean diamond. The correspondence with the theoretical line of $T_{2}$ (Ref. 5) is very important to elucidate the dephasing mechanism and to make $T_{2}$ longer for quantum information devices ${ }^{4}$ and ultraprecise magnetometry. ${ }^{6,7}$ Furthermore, the results show that the threshold $\left(\sim 10^{4}\right.$ operation) for quantum error-correction schemes ${ }^{27}$ can be exceeded even in ${ }^{13} \mathrm{C}$ enriched diamond at room temperature with typical single-qubit flip of several ns.

This work is supported by the EU (QAP, EQUIND, NANO4DRUGS, NEDQIT), DFG (Contracts No. SFB/TR21 and No. FOR730), JST-DFG program KAKENHI (Grant No. 20760006), and the Landesstiftung BW. V.J. acknowledges support by the Humboldt Stiftung. We thank H. Kanda for providing $100 \%{ }^{13} \mathrm{C}$ diamond.
${ }^{1}$ F. Jelezko, T. Gaebel, I. Popa, M. Domhan, A. Gruber, and J. Wrachtrup, Phys. Rev. Lett. 93, 130501 (2004).

${ }^{2}$ M. V. Gurudev Dutt, L. Childress, L. Jiang, E. Togan, J. Maze, F. Jelezko, A. S. Zibrov, P. R. Hemmer, and M. D. Lukin, Science 316, 1312 (2007).

${ }^{3}$ P. Neumann, N. Mizuochi, F. Rempp, P. Hemmer, H. Watanabe, S. Yamasaki, V. Jacques, T. Gaebel, F. Jelezko, and J. Wrachtrup, Science 320, 1326 (2008).

${ }^{4}$ L. Jiang, J. M. Taylor, A. S. Sorensen, and M. D. Lukin, Phys. Rev. A 76, 062323 (2007);

${ }^{5}$ J. R. Maze, J. M. Taylor, and M. D. Lukin, Phys. Rev. B 78, 094303 (2008).

${ }^{6} \mathrm{G}$. Balasubramanian et al., Nature (London) 455, 648 (2008).

${ }^{7}$ J. R. Maze et al., Nature (London) 455, 644 (2008).

${ }^{8}$ L. Childress, M. V. Gurudev Dutt, J. M. Taylor, A. S. Zibrov, F. Jelezko, J. Wrachtrup, P. R. Hemmer, and M. D. Lukin, Science 314, 281 (2006).

${ }^{9}$ S. Takahashi, R. Hanson, J. van Tol, M. S. Sherwin, and D. D. Awschalom, Phys. Rev. Lett. 101, 047601 (2008).

${ }^{10}$ R. Hanson, V. V. Dobrovitski, A. E. Feiguin, O. Gywat, and D. D. Awschalom, Science 320, 352 (2008).

${ }^{11}$ A. Gruber, A. Dräbenstedt, C. Tietz, L. Fleury, J. Wrachtrup, and C. von Borczyskowski, Science 276, 2012 (1997).

${ }^{12}$ Jan Isberg, Johan Hammersberg, Erik Johansson, Tobias Wikström, Daniel J. Twitchen, Andrew J. Whitehead, Steven E. Coe, and Geoffrey A. Scarsbrook, Science 297, 1670 (2002).

${ }^{13}$ N. Mizuochi, et al., J. Appl. Phys. 101, 103501 (2007).

${ }^{14}$ K. Nakamura, S. Yamashita, T. Tojo, M. Mitsuishi, K. Kataoka, and M. Yoshimoto, Diamond Relat. Mater. 16, 1765 (2007).
${ }^{15}$ N. B. Manson, J. P. Harrison, and M. J. Sellars, Phys. Rev. B 74, 104303 (2006).

${ }^{16}$ J. H. N. Loubser and J. A. van Wyk, Diamond Research (Suppl. to Ind. Diamond Rev.) pp. 11-14 (1977).

${ }^{17}$ A. Gali, M. Fyta, and E. Kaxiras, Phys. Rev. B 77, 155206 (2008).

${ }^{18}$ See EPAPS Document No. E-PRBMDO-80-R14928 for the assigned sites of ${ }^{13} \mathrm{C}$ at the third shell. For more information on EPAPS, see http://www.aip.org/pubservs/epaps.html.

${ }^{19}$ A. Schweiger et al., Principles of Pulsed EPR (Oxford University Press, New York, 2001).

${ }^{20}$ M. Mehring, J. Mende, and W. Scherer, Phys. Rev. Lett. 90, 153001 (2003).

${ }^{21}$ C. F. Roos, G. P. T. Lancaster, M. Riebe, H. Haffner, W. Hansel, S. Gulde, C. Becher, J. Eschner, F. Schmidt-Kaler, and R. Blatt, Phys. Rev. Lett. 92, 220402 (2004).

${ }^{22}$ R. Ernst et al., Principles of NMR in One and Two Dimensions (Oxford Press, New York, 1989), Chap. 5.

${ }^{23}$ W. Kohn, Solid State Physics (Academic Press, New York, 1957), Vol. 5, p. 257.

${ }^{24}$ E. Abe, A. Fujimoto, J. Isoya, S. Yamasaki, and K. Itoh, arXiv:cond-mat/0512404 (unpublished).

${ }^{25}$ J. H. Van Vleck, Phys. Rev. 74, 1168 (1948).

${ }^{26} \mathrm{G}$. Balasubramanian et al., Nature Mater. 8, 383 (2009).

${ }^{27}$ D. D. Awshalom et al., Semiconductor Spintronics and Quantum Computation (Spring-Verlag, Berlin, 2002).

${ }^{28}$ V. Jacques, P. Neumann, J. Beck, M. Markham, D. Twitchen, J. Meijer, F. Kaiser, G. Balasubramanian, F. Jelezko, and J. Wrachtrup, Phys. Rev. Lett. 102, 057403 (2009). 